\title{
Docking study on Mycobacterium tuberculosis receptors AccD5 and PKS18 with selected phytochemicals
}

\author{
Dr. C. Anchana Devi \\ Assistant Professor, $P G \&$ Research Department of Biotechnology, Women's Christian College, \\ Chennai 600005
}

\begin{abstract}
Epidemiological studies have indicated that 1.7 billion people, as much as one third of the world's population, are infected with Mycobacterium tuberculosis, the causative agent of human tuberculosis (TB). This pathogen is responsible for more human deaths than any other single infectious agent throughout the centuries of human history. Considering the world-wide TB problems, there is an urgent need to develop relatively inexpensive new drugs to treat this deadly disease. Natural products isolated from plants have played an important role in discovery of drugs against infectious diseases. In the present study, three ligand molecules (basically secondary metabolites) which were commonly present in the plants were docked with the selected Mycobacterium receptor AccD5 and PKS18. Among them Phytol had a significant inhibitory activity with the receptor AccD5 binding to the pocket (GLY 241-GLY 242) forming hydrogen bonds at a very low energy value, thus forming a stable complex.The active compounds were found to be diterpene alcohol and isomers of diterpene alcohol. These molecules had a good number of conformations showing the flexible behavior of the ligand. The total energy of the receptor and ligand complexes has also been calculated.

Key Words: Mycobacterium tuberculosis, Molecular docking, Phytol, AccD5.
\end{abstract}

\section{Introduction}

Microbial infections are a growing problem in contemporary medicine, yet only a few antimicrobial agents are used in clinical practice. Mycobacterium tuberculosis (MTB) is a pathogenic bacterial species in the genus Mycobacterium and the causative agent of most cases of tuberculosis [1]. Tuberculosis (TB), a lung infection and is one of the contagious and deadly diseases which have added to the woes of the mankind. The main reason for the widespread of this disease is the population growth, emergence of multi-drug resistant TB strains, financial burden in the developing countries and unsuccessful attempt to synthesize a new drug with novel mechanism of action.

Although one possible long-term solution to the problem is a better vaccine, in the short term, the major reliance will be on chemotherapy [2] requiring the development of novel, effective and non-toxic antitubercular agents [3-5]. The identification of novel target sites will also be needed to circumvent the problems associated with the increasing occurrence of multi-drug resistant strains. To do this, biochemical pathways specific to the mycobacteria and related organisms' disease cycle must be better understood. Many unique metabolic processes occur during the biosynthesis of mycobacterial cell wall components [6]. One of these attractive targets for the rational design of new antitubercular agents are the mycolic acids and the major components of the cell wall of Mycobacterium tuberculosis [7].

The two type III PKS proteins, PKS18 and PKS11, from Mycobacterium tuberculosis that are involved in the biosynthesis of long-chain $\alpha$-pyrones to construct the mycolic acid and multimethyl-branched fatty acid was found uniquely in the cell envelope of pathogen Mycobacterium. These long unusual fatty acids are essential for the survival of virulence and antibacterial resistance of Mycobacterium tuberculosis. In Mycobacterium tuberculosis, Acyl-CoA carboxylase carboxyltransferase (ACCase) consists of three subunits namely $\alpha, \beta$ and $\varepsilon$ [7] AccD5 plays major role in cell envelope lipid biosynthesis and its disruption lead to pathogen death.

In molecular modeling, docking is a method which predicts the preferred orientation of one molecule to a second when bound to each other to form a stable complex. Knowledge of the preferred orientation in turn may be used to predict the strength of association or binding affinity between two molecules using for example scoring functions $[8,9]$. The protein structure and a database of potential ligands serve as inputs to a docking program. Molecular docking algorithms fit molecules together in complementary fashions. The technique has attracted increasing attention as a way to predict the geometries of bimolecular complexes [10]. Most of docking programs in use account for a flexible ligand, and several are attempting to model a flexible protein receptor. The present study has been carried out to test the efficiency of the naturally occurring plant compounds against tuberculosis AccD5 and PKS18 using MOLECULAR DOCKING STUDIES with the objective to find potential drug targets. 


\section{Ligands selected for the study:}

\section{Methodology}

The set of ligand molecules selected for the study were commonly present in all the three plants Ficusreligiosa, SaracaasocaandTylophoraindica in this work include Phytol (CID 145386) and its structurally similar compounds 3, 7, 11, 15 - tetramethyl-2-hexadecen-1-ol (CID 5366244) and n-hexadecenoic acid (CID 57-10-3). These ligand molecules were retrieved from NCBI-PubChem Compound database (http//pubchem.ncbi.nlm.nih.gov/) Wheeler.(2007 ). The structure of these compounds is shown in Fig. 1.

\section{Receptors Selected for the study:}

The three dimensional structure of protein receptors PKS 18(PDB 1TED) and AcccD5 (PDB ID2A7S) Fig. 2 were obtained from Protein data bank which is present in the Mycobacterium tuberculosis. The receptor molecules were obtained from Protein Data Bank (PDB) (http//www.rcsb.org/pdb/home/home.do)The energy of the ligand molecules and target PDI were minimized in Steepest Descent followed by Conjugate Gradient method using Accelrys Discovery Studio (Version 1.7, Accelrys Software Inc.), the most comprehensive suite of modeling and simulation solutions for drug discovery was available. Each of the minimization method was carried out using CHARMm force field.

In order to carry out the docking simulation, we used the AutoDock 4.0 suite as molecular-docking tool [7] it is the suitable software for performing automated docking of ligands to their macromolecular receptors. Typically, the ligands are substrates or drug candidates and the macromolecule is a protein of known three dimensional structures. In this docking simulation, we used semi-flexible docking protocol, in which the target protein PDI was kept as rigid. The ligands being docked were kept flexible, in order to explore an arbitrary number of torsional degrees of freedom in addition to the six spatial degrees of freedom spanned by the translational and rotational parameters. The Graphical User Interface program "Auto- Dock Tools" was used to prepare, run and analyze the docking simulations. The atom charges, salvation parameters and polar hydrogens were added into the receptor PDB file for the preparation of protein in docking simulation. This PDI enzyme structure does not have any water molecules and/or ligands to remove from its PDB file and make a free receptor.

Since ligands are not peptides, Gasteiger charge was assigned and then nonpolar hydrogens were merged. The rigid roots of each ligand were defined automatically instead of picking manually. The amide bonds were made non-rotatable. All rotatable dihedrals in the ligands were assigned with the help of auxiliary program Auto-Tors and were allowed to rotate freely. AutoDock requires pre-calculated grid maps, one for each atom type, present in the ligand being docked and its stores of potential energy arising from the interaction with macromolecule. This grid must surround the region of interest in the macromolecule. Therefore, the grid was centered in the catalytic active region of redoxactive motif and includes all amino acid residues that surround active site. The grid box size was set at 40,40, and $40 \mathrm{~A}^{\circ}$ (x, y, and $\mathrm{z}$ ), though it was changed depending on the ligand size. AutoGrid 4.0 Program, supplied with AutoDock 4.0 was used to produce grid maps. The spacing between grid points was 0.375 angstroms.

The Lamarckian Genetic Algorithm (LGA) was chosen to search for the best conformers. During the docking process, a maximum of 10 conformers was considered for each compound. Maximum number of energy evaluation was set to 250000 , maximum number of generations 1000 , maximum number of top individual that automatically survived set to 1 , mutation rate of 0.02 , crossover rate of 0.8 , Step sizes were $0.2 \AA$ for translations, $5.0^{\circ}$ for quaternion's and $5.0^{\circ}$ for torsions. Cluster tolerance $0.5 \mathrm{~A}^{\circ}$, external grid energy 1000.0 , max initial energy 0.0, max number of retries 10000 and 10 LGA runs were performed. All the Auto Dock docking runs were performed in Intel Pentium PD-925 CPU @ 3.0 GHz of HCL info system origin, with 2 GB DDR RAM. Auto- Dock 4.0 was compiled and run under Windows XP operating system and the results were obtained and interpreted.

\section{Result And Discussion}

The phytol, n-hexadecenoic acid and 3, 7, 11, 15-tetramethyl 2-hexadecen-1-ol acts as ligands and docked with the selected receptor of Mycobacterium tuberculosis PKS 18 and AccD5. For years, docking has been a powerful tool for medicinal chemists, allowing the rapid and inexpensive identification of a pool of potential protein inhibitors [11-13]. Lately, docking simulations have been used, in the field of structural biology, for a different purpose: the identification of plausible substrates of proteins of unknown function [14$18]$.

The PKS 18 docked with phytol, these two rounds of virtual screening were performed and finally Hydrogen molecules were obtained, which bound at different binding pockets, mainly at THR 529 and Glu 569 (Fig. 3 a, b). The PKS 18 homology model shows remarkable constriction in the active site cavity. The steric bulk from three active site residue, significantly affects the active site volume, therefore PKS 18 protein resembles 2PS and may catalyses biosynthesis of long chain fatty acids. Another main reason of selection of 
PKS 18 is found to be remarkable substrate specificity was studied by Sankaranarayananet al. [19]. This study clearly suggested that PKS 18 protein can extend a long chain acyl CoA substrate by using specifically malonyl $\mathrm{CoA}$ as extends revealing the specificity of PKS 18 protein against Mycobacterium tuberculosis.

The ligands are screened for their ability to dock within the catalytic triad of AccD5 and to find those chemical compounds which can inhibit the activity of AccD5 and for these two rounds of virtual screening are performed and finally got Hydrogen molecules that bound at different binding pockets, mainly at GLY 241 and GLY 242 (Fig. 4). These molecules have multiple conformations. The molecular surfaces are generated which are used to cluster spheres. Site points are constructed within $10 \AA$ of the ligand using the sphere generation accessory program of DOCK.

In the present study revealed phytol having the desired potential to block the active site of the receptor AccD5. The docking molecule (phytol) exhibited a low energy value of -11.03282 than with PKS 18 , the latter having comparatively a lower energy value of -1.29 (Table 1 ). The other two ligands $n$-hexadecenoic acid and 3, 7, 11, 15-tetramethyl 2-hexadecen-1-ol showed negative action against the selected Mycobacterium tuberculosis receptors.

Fig. 1 Structure of bioactive compounds (Phytochemicals)selected for the study from plant sources

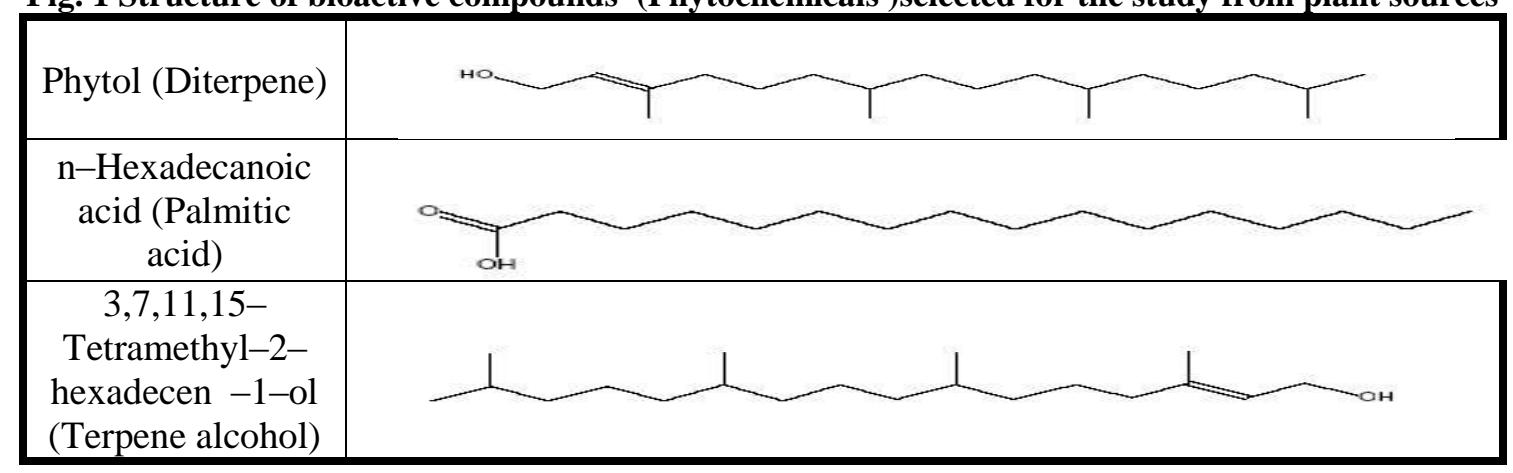

Table 1 Active sites of selected receptors PKS 18 and AccD5

\begin{tabular}{|c|c|c|c|c|}
\hline $\begin{array}{c}\text { Receptor } \\
\text { Compound }\end{array}$ & Active sites & $\begin{array}{l}\text { Binding } \\
\text { Residue }\end{array}$ & Energy value & $\begin{array}{c}\text { Inhibition } \\
\text { Constant Value }\end{array}$ \\
\hline PKS 18 & $\begin{array}{l}\text { ASN 360, THR 392, LYS 394, } \\
\text { PHE 423, GLU 425, TRP 426, } \\
\text { MET 436, LYS 437, TYR 438, } \\
\text { SER 439, ASN 441, TRP 477, } \\
\text { ASN 478, SER 514, MET 515, } \\
\text { THR 516, LEU 517, GLY 527, } \\
\text { LYS 528, THR 529, GLY 530, } \\
\text { THR 531, GLY 532, ILE 533, } \\
\text { GLU 538, PHE 543, ASN 567, } \\
\text { GLY 568, GLY 569, GLN } \\
\text { 572. }\end{array}$ & $\begin{array}{l}\text { THR 529- } \\
\text { GLU 569 }\end{array}$ & -1.29 & 113.95 \\
\hline AccD5 & GLY 240 GLY 241- GLY 242 & $\begin{array}{l}\text { GLY } 241- \\
\text { GLY } 242\end{array}$ & -11.03282 & -6.82 \\
\hline
\end{tabular}

Fig. 2 PKS 18 (PDB 1TED)

AccD5 (PDB ID2A7S)
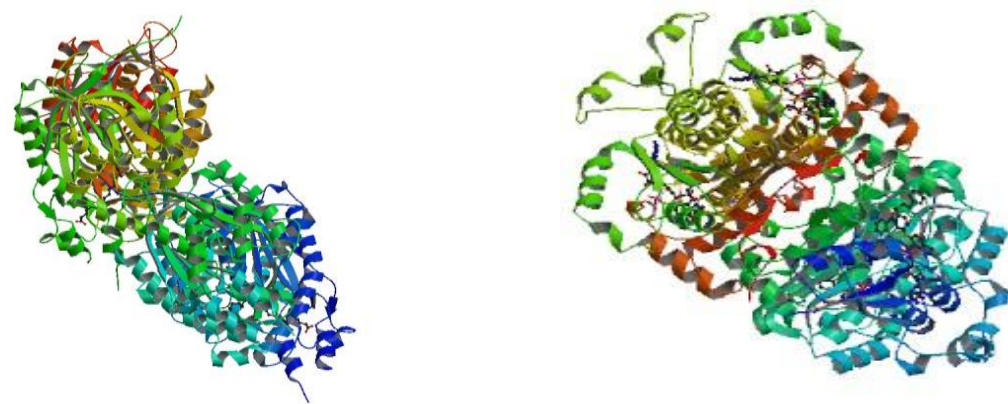
Fig. 3 Formation of Hydrogen bond between THR 529 - GLU 569 of the Phytol compound with PKS 18 receptor
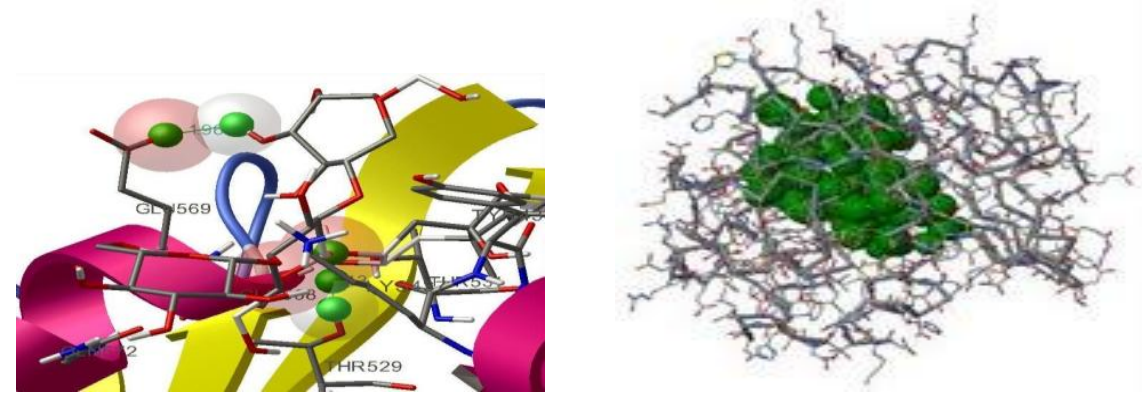

Fig. 4 Formation of Hydrogen bond between GLY 241-GLY242 of the Phytol compound with the AccD5 receptor
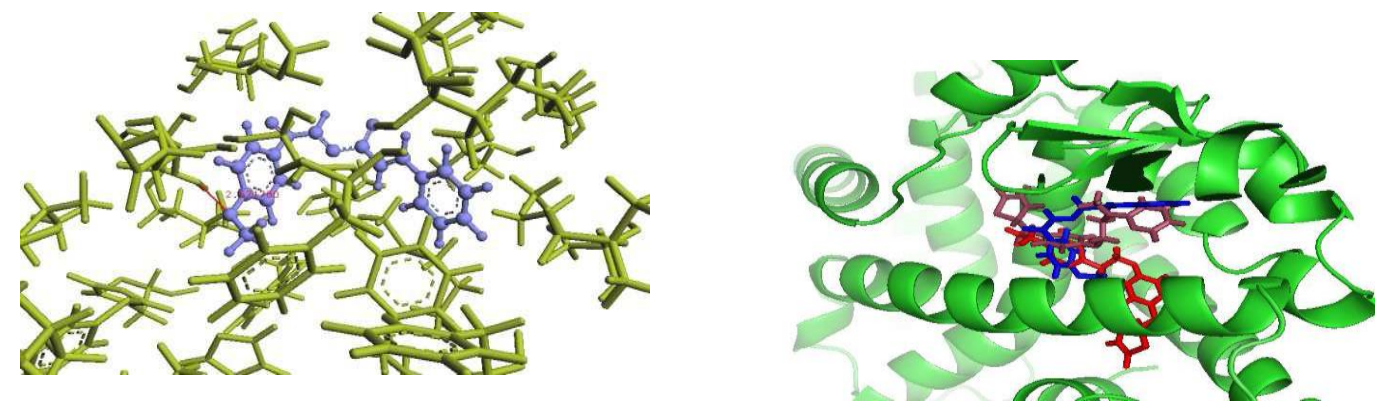

\section{References}

[1] Ryan, K.J. and Ray, C.G. (2004). (Eds.), Sherris Medical Microbiology, fourth ed. McGraw Hill, ISBN 0-8385-8529-9.

[2] Tomioka, H. and Namba, K. (2006). Development of antitubercular drugs: current status and future prospects, Kekkaku (Japanese Journal). 81(12):753-774.

[3] Berning, S.E. (2001). The role of fluoroquinolones in tuberculosis today, Drugs. 61:9-18.

[4] Reddy, V.M., Nadadhur, G., Daneluzzi, D.D., Osullivan, J.F. and Gangadharam, P.R.J. (1996). Antituberculosis activities of clofazimine and its new analogs B4154 and B4157, Antimicrob. Agents Chemother. 40:633-636.

[5] Barry, C.E. (1997). New horizons in the treatment of tuberculosis, Biochem.Pharmacol. 54:1165-1172.

[6] Pasquato, K.F.M. and Ferreira, E.I. (2001). An approach for the rational design of new antitubercular agents, Curr. Drug Targets. 2:427-437.

[7] Diacovich, L., Mitchell, D., Pham, H., Gago, G., Melgar, M. M., Khosla, C., Gramajo, H. and Tsai, S.C. (2004). Crystal structure of the beta-subunit of acyl-CoA carboxylase: structure-based engineering of substrate specificity. http://www.ncbi.nlm.nih.gov/pubmed?term=\%22Diacovich \%22\%5BAuthor\%5DBiochemistry. 43:14027-36.

[8] Ewing, T., Makino, S., Skillman, A. and Kuntz, I. (2001).DOCK 4.0: search strategies for automated molecular docking of flexible molecule databases. J Comput Aided Mol. Des. 15 (5): 411-28.

[9] Bursulaya, B., Totrov, M., Abagyan, R. and Brooks, C. (2003).Comparative Study of Several Algorithms For Flexible Ligand Docking.J Comput Aided Mol Des.17: 755-63.

[10] Irawin D. Kuntz, Elaine C. Meng, and Brain K. Shoichet (1994). Acc. Chem. Res. 27 (5):117-123.

[11]. Jorgensen, W.L. (2004).The many roles of computation in drug discovery. Science. 303:1813-1818

[12]. Brooijmans, N. and Kuntz, I.D. (2003).Molecular recognition and docking algorithms.Annu. Rev. Biophys. Biomol. Struct. 32:335373.

[13] Shoichet, B.K., McGovern, S.L., Wei, B. and Irwin, J.J. (2002). Lead discovery using molecular docking. Curr. Opin. Chem. Biol. 6:439-446.

[14] Hermann, J.C., Ghanem, E., Li, Y., Raushel, F.M., Irwin, J.J. and Shoichet, B.K. (2006). Predicting substrates by docking highenergy intermediates to enzyme structures. J. Am. Chem. Soc. 128:15882-15891.

[15] Kalyanaraman, C., Bernacki, K. and Jacobson, M. P. (2005). Virtual screening against highly charged active sites: identifying substrates of alpha-beta barrel enzymes. Biochemistry. 44: 2059-2071.

[16] Bernacki, K., Kalyanaraman, C. and Jacobson, M.P. (2005). Virtual ligand screening against Escherichia coli dihydrofolatereductase: improving docking enrichment using physics-based methods. J. Biomol. Screening. 10:675-681.

[17] Macchiarulo, A., Nobeli, I. and Thornton, J.M. (2004). Ligand selectivity and competition between enzymes in silico. Nat. Biotechnol. 22:1039-1045.

[18] Hermann, J.C., Marti-Arbona, R., Fedorov, A.A., Fedorov, E., Almo, S.C., Shoichet, B.K. and Raushel, F.M. (2007). Structurebased activity prediction for an enzyme of unknown function. Nature. 448:775-779.

[19] Sankaranarayanan, R., Saxena, P., Marathe, U.B., Gokhale, R.S., Shanmugam, V.M. and Rukmini, R. (2004). A novel tunnel in mycobacterial type III polypeptide synthase reveals the structural basis for generating diverse metabolites.Nat StructMol Biol. 11(9):894-900. 